- Original Article

\title{
Serum Alanine Aminotransferase Level as a Risk Factor for Coronary Heart Disease Prediction in Koreans: Analysis of the Korea National Health and Nutrition Examination Survey ( $\mathrm{V}-1$, 2010 and V-2, 2011)
}

\author{
Kiyoung Kim, Dongsun Kim, Kyu-Nam Kim* \\ Department of Family Practice and Community Health, Ajou University School of Medicine, Suwon, Korea
}

\begin{abstract}
Background: The blood level of alanine aminotransferase (ALT) is associated with increased coronary heart disease (CHD) risk. However, its use as an independent factor for CHD risk prediction remains unclear in Asian populations. The purpose of this study was to examine the association between serum ALT levels and CHD risk in Koreans.

Methods: This was a cross-sectional study using data from the Korea National Health and Nutrition Examination Survey (V-1, 2010 and V-2, 2011). The ALT levels of 3,215 individuals were analyzed. The Framingham Risk Score (FRS) modified by the National Cholesterol Education Program Adult Treatment Panel III (NCEP ATP III) was used to compute the 10-year CHD risk prediction.

Results: Positive correlations were established between log-transformed ALT concentration and FRS ( $\mathrm{r}=0.433$, $\mathrm{P}<0.001$ ). After adjusting for body mass index, low-density lipoprotein cholesterol, the amount of alcohol intake, and gamma-glutamyl transferase, the odds ratio (95\% confidence interval) for intermediate or greater risk of 10year CHD prediction (10-year risk $\geq 10 \%$ ) for the lowest quartile of participants was 2.242 (1.405-3.577) for the second quartile, 2.879 (1.772-4.679) for the third quartile, and 3.041 (1.789-5.170) for the highest quartile.

Conclusion: In Koreans, a higher serum ALT concentration was significantly correlated with 10-year CHD risk prediction according to NCEP ATP III guidelines.
\end{abstract}

Keywords: Alanine Aminotransferase; Coronary Disease; Framingham Risk Score

Received: May 22, 2017, Revised: September 5, 2017, Accepted: October 12, 2017

*Corresponding Author: Kyu-Nam Kim https://orcid.org/0000-0002-1213-5004

Tel: +82-31-219-5324, Fax: +82-31-219-5218, E-mail: ktwonm@hanmail.net 


\section{INTRODUCTION}

Alanine aminotransferase (ALT) catalyzes the transfer of amino groups to form hepatic metabolite oxaloacetate, an intracellular enzyme. ${ }^{1)}$ ALT is concentrated in hepatocytes. An increased ALT level indicates liver injury. Thus, ALT has been widely used in clinical practice to evaluate liver function. ${ }^{2-5)}$ In addition, ALT concentration has been used as a marker of non-alcoholic fatty liver disease (NAFLD). It is positively correlated with liver fat measured by magnetic resonance proton spectroscopy. ${ }^{6,7)}$ Several studies have demonstrated that NAFLD is a component of metabolic syndrome (MetS) ${ }^{8,9)}$ associated with diabetes mellitus (DM) ${ }^{10)}$ On the basis of these findings, some researchers have reported that ALT is associated with DM and MetS. ${ }^{6,11)}$ Taken together, these data suggest that an elevated ALT level as a biomarker of hepatic steatosis can play a role in the pathogenesis of DM and MetS, probably by contributing to the development of hepatic insulin resistance. Subjects with features of DM and MetS are reported to be at risk of developing coronary heart disease (CHD). ${ }^{12)} \mathrm{A}$ prospective study reported that an increased ALT level predicts CHD events independently of traditional cardiovascular risk factors or components of MetS in Caucasian men and women. ${ }^{13)}$ Despite the clinical impact of increased ALT levels in Western countries, little is known about the usefulness of ALT levels in the prediction of CHD risk in Asian populations.

In view of these observations, the objective of this study was to determine the predictive value of using the ALT level for CHD risk prediction in Korean men and women without DM or CHD. The Framingham Risk Score (FRS) modified by the National Cholesterol Education Program Adult Treatment Panel III (NCEP ATP III) guidelines and data from the Korea National Health and Nutrition Examination Surveys
(KNHANES) from 2010 to 2011 were used in this study to compute the risk of $\mathrm{CHD}$.

\section{METHODS}

\section{Study Subjects}

In this cross-sectional study, data were taken from KNHANES (KNHANES V-1, 2010 and V-2, 2011), a national survey representing the general Korean population. KNHANES provides comprehensive information on the health status, nutritional status, sociodemographics, and health behavior of Koreans in 600 national districts. It has been conducted by the Korea Centers for Disease Control and Prevention since 1998.

We excluded 5,605 of 8,983 people due to missing data on FRS, smoking, or alcohol history. In addition, we excluded 163 subjects with DM, CHD, positive test results for antibody to hepatitis B surface antigen, antibody to hepatitis B virus core antigen or hepatitis $C$ virus, hepatobiliary disease, and patients who were taking lipid-lowering drugs and drugs that might influence liver function. Finally, a total of 3,215 subjects were included in this study. Weekly alcohol consumption was calculated and converted into grams of ethanol intake. ${ }^{14)}$ Subjects who had been smoking cigarettes regularly during the year before the survey were considered current smokers.

\section{Measurements}

After overnight fasting, a venous blood sample was obtained between 8:00 and 10:00 AM to measure ALT and liver enzyme, total cholesterol, triglyceride, high-density lipoprotein (HDL) cholesterol, and low-density lipoprotein (LDL) cholesterol levels. Each subject fasted for more than 10 hours before blood collection.

Table 1. General characteristics of study subjects according to serum ALT grading

\begin{tabular}{|c|c|c|c|c|c|c|}
\hline \multirow{2}{*}{ Characteristic } & \multirow{2}{*}{ Total } & \multicolumn{4}{|c|}{ ALT grading } & \multirow{2}{*}{ P for trend } \\
\hline & & $Q 1^{*}(n=836)$ & Q2 ( $\mathrm{n}=732)$ & Q3 $(n=821)$ & Q4 (n=826) & \\
\hline Age $(y)$ & $43.3 \pm 0.5$ & $37.6 \pm 0.5$ & $44.8 \pm 0.6$ & $46.4 \pm 0.5$ & $44.4 \pm 0.5$ & $<0.001$ \\
\hline Body mass index (kg/m²) & $23.5 \pm 0.1$ & $21.7 \pm 0.1$ & $23.1 \pm 0.1$ & $23.9 \pm 0.1$ & $25.2 \pm 0.1$ & $<0.001$ \\
\hline Alcohol (g/wk) & $41.4 \pm 2.2$ & $16.2 \pm 1.2$ & $29.4 \pm 3.1$ & $49.1 \pm 3.2$ & $71.1 \pm 5.2$ & $<0.001$ \\
\hline Hypertension (\%) & 8.6 & 4.8 & 7.9 & 9.8 & 12.9 & $<0.001$ \\
\hline Systolic blood pressure (mm Hg) & $113.7 \pm 2.9$ & $101.5 \pm 1.8$ & $109.1 \pm 2.6$ & $119.8 \pm 4.7$ & $124.6 \pm 4.9$ & $<0.001$ \\
\hline Diastolic blood pressure (mm Hg) & $76.9 \pm 0.4$ & $72.7 \pm 0.3$ & $75.7 \pm 0.4$ & $78.2 \pm 0.4$ & $81.1 \pm 0.4$ & $<0.001$ \\
\hline Total cholesterol (mg/dL) & $169.3 \pm 2.1$ & $176.2 \pm 1.1$ & $109.1 \pm 2.6$ & $192.1 \pm 1.2$ & $199.9 \pm 1.4$ & $<0.001$ \\
\hline High-density lipoprotein (mg/dL) & $49.5 \pm 0.6$ & $55.1 \pm 0.5$ & $51.4 \pm 0.6$ & $48.4 \pm 0.6$ & $43.1 \pm 0.6$ & $<0.001$ \\
\hline Low-density lipoprotein (mg/dL) & $114.2 \pm 1.1$ & $103.6 \pm 1.1$ & $114.2 \pm 1.1$ & $116.7 \pm 1.1$ & $122.2 \pm 1.2$ & $<0.001$ \\
\hline Triglyceride (mg/dL) & $130.5 \pm 3.1$ & $88.5 \pm 1.8$ & $109.1 \pm 2.6$ & $141.8 \pm 4.7$ & $182.6 \pm 4.9$ & $<0.001$ \\
\hline Current smoking (\%) & 25.1 & 13.5 & 20.9 & 28.3 & 37.8 & $<0.001$ \\
\hline Aspartate aminotransferase (IU/L) & $21.5 \pm 0.3$ & $15.5 \pm 0.1$ & $18.6 \pm 0.1$ & $21.1 \pm 0.1$ & $30.8 \pm 0.7$ & $<0.001$ \\
\hline Gamma-glutamyl transferase (IU/L) & $32.9 \pm 0.5$ & $15.3 \pm 0.3$ & $21.2 \pm 0.5$ & $30.9 \pm 0.9$ & $64.4 \pm 2.7$ & $<0.001$ \\
\hline Total Framingham point scores & $4.4 \pm 0.2$ & $0.8 \pm 0.2$ & $2.6 \pm 0.4$ & $6.3 \pm 0.3$ & $7.9 \pm 0.2$ & $<0.001$ \\
\hline $10-Y e a r$ coronary heart disease risk & $3.7 \pm 0.2$ & $1.4 \pm 0.1$ & $3.1 \pm 0.2$ & $4.6 \pm 0.2$ & $5.8 \pm 0.2$ & $<0.001$ \\
\hline
\end{tabular}

Values are presented as mean \pm standard error after data weighting in complex sample analysis, unless otherwise stated. Analysis of variance trend analysis was used for continuous variables, and chi-square tests were used for categorical variables.

ALT, alanine aminotransferase.

*Quartile: Q1, 1st quartile; Q2, 2nd quartile; Q3, 3rd quartile; Q4, 4th quartile. 
Serum ALT and AST levels, and lipid levels were assayed using Hitachi Automatic Analyzer 7600 (Hitachi, Tokyo, Japan). Body mass index (BMI) was calculated as weight $(\mathrm{kg})$ divided by height squared $\left(\mathrm{m}^{2}\right)$. Blood pressure (BP) was measured with a standard mercury manometer. The participant maintained a sitting position for 5 minutes before the measurement. The average measurement was recorded. Hypertension (HTN) was defined as a systolic BP (SBP) $\geq 140 \mathrm{~mm} \mathrm{Hg}$, diastolic $\mathrm{BP} \geq 90 \mathrm{~mm} \mathrm{Hg}$, or use of antihypertensive medication.

\section{Statistical Analyses}

For weighting assessment of KNHANES data, complex sample analysis was employed. Subjects were grouped into quartiles according to levels of serum ALT. Analysis of variance (ANOVA) trend analysis using polynomial contrasts was adapted to perform tests for trends. Distribution of ALT and gamma-glutamyl transferase (GGT) values were right-skewed. Thus, a natural log-transformation was applied. To determine the relationship between serum ALT activity and individual components of FRS, Spearman's correlation analysis was performed.

FRS was calculated on the basis of the following six coronary risk factors from the NCEP ATP III algorithm: age, sex, HDL cholesterol, total cholesterol, SBP, and smoking habits. Age, BP, and cholesterol levels were grouped according to their values. To predict the risk of coronary disease events (myocardial infarction or CHD death) over the next 10year period for adults aged 20 and older who had no heart disease or diabetes, Framingham risk equations were employed. Subjects were divided into three groups: low risk ( $<10 \%$ risk of developing CHD event over the next 10 years), intermediate risk ( $10 \%-20 \%$ risk), and high risk ( $>20 \%$ risk). In the intermediate- or greater-risk group of 10-year predicted CHD, adjusted logistic regression analyses were performed taking into consideration BMI, weekly alcohol intake, log-transformed GGT, and LDL cholesterol. Data are expressed as mean \pm standard error. $\mathrm{P}<0.05$ was considered statistically significant. In Table 1 , ANOVA trend analysis was used for continuous variables, and chi-square tests were used for categorical variables. Smoking status was classified as either current smoker or nonsmoker. Data were analyzed using PASW SPSS ver. 18.0 (SPSS Inc., Chicago, IL, USA).

\section{RESULTS}

This study included a total of 3,215 subjects. Of these, 1,541 were male and 1,674 were female. Distribution of age was as follows: 20-29 years,
701; 30-39 years, 730 ; $40-49$ years, 688 ; 50-59 years, $584 ; 60-69$ years, 439; and >70 years, 73 . These subjects were divided into four groups according to ALT levels. Ranges for the 1st, 2nd, 3rd, and 4th quartiles of serum ALT values were 3-12, 13-16, 17-24, and 25-156 IU/L, respectively. General characteristics of the study participants are summarized in Table 1. Participants in higher ALT quartiles had more general CHD risk factors such as HTN; elevated BMI, triglyceride, and LDL cholesterol; higher alcohol consumption; and current smoking. In addition, higher quartiles of serum ALT concentrations were significantly associated with increasing risk of 10 -year CHD prediction ( $\mathrm{P}$ for trend $<0.05)$.

The correlation between log-transformed ALT and 10-year CHD risk was significant $(\mathrm{r}=0.433, \mathrm{P}<0.001)$ (Table 2). Individual risk factor scores according to age, smoking, total cholesterol, HDL-cholesterol, and SBP were also significantly correlated with log-transformed ALT $(\mathrm{P}<0.05$ for all).

Odds ratios (ORs) for intermediate or greater risk of 10-year CHD prediction for CHD according to quartiles of serum ALT are shown in Table 3. The 10-year CHD risk was significantly associated with increasing quartile of serum ALT ( $\mathrm{P}$ for trend $<0.05$ ). Compared to individuals with the lowest quartile of serum ALT, the non-adjusted OR (95\% confidence interval [CI]) was 2.753 (1.720-4.405) for the second quartile, 4.381 (2.777-6.913) for the third quartile, and 7.602 (4.83211.961) for the fourth quartile. After adjusting for BMI, the amount of alcohol intake, log-transformed GGT, and LDL cholesterol, the OR (95\% CI) for intermediate or greater risk of 10 -year CHD prediction in the lowest quartile of participants was 2.242 (1.405-3.577) for the second quartile, 2.879 (1.772-4.679) for the third quartile, and 3.041 (1.789-5.170) for the highest quartile. In addition, the OR for intermediate or greater risk of 10-year CHD prediction showed an increased

Table 2. Spearman's rank correlation coefficients for the relationship between individual component or total Framingham risk score and log-transformed alanine aminotransferase

\begin{tabular}{lcc}
\hline \multicolumn{1}{c}{ Variable } & Correlation coefficient & P-value \\
\hline Age score & 0.122 & $<0.001$ \\
Total cholesterol score & 0.126 & $<0.001$ \\
Smoking score & 0.212 & $<0.001$ \\
Systolic blood pressure score & 0.144 & $<0.001$ \\
High-density lipoprotein cholesterol score & 0.281 & $<0.001$ \\
Total Framingham point score & 0.310 & $<0.001$ \\
10-Year coronary heart disease risk & 0.433 & $<0.001$ \\
\hline
\end{tabular}

Table 3. Odds ratio of intermediate or greater 10-year risk of coronary heart disease (10-year risk $\geq 10 \%)$ by ALT level

\begin{tabular}{|c|c|c|c|c|c|}
\hline \multirow{2}{*}{ Model } & \multicolumn{4}{|c|}{ ALT } & \multirow{2}{*}{$P$ for trend } \\
\hline & $Q 1 *(n=836)$ & $Q 2(n=732)$ & Q3 (n=821) & Q4 (n=826) & \\
\hline Model 1 & 1.00 & $2.753(1.720-4.405)$ & $4.381(2.777-6.913)$ & 7.602 (4.832-11.961) & $<0.001$ \\
\hline Model 2 & 1.00 & $2.242(1.405-3.577)$ & $2.879(1.772-4.679)$ & $3.041(1.789-5.170)$ & $<0.001$ \\
\hline
\end{tabular}

Values are presented as odds ratio (95\% confidence interval). The multivariate logistic regression model was used after data weighting in complex sample analysis. Model 1 : unadjusted; model 2: after adjustment for body mass index, amount of alcohol consumption, log-transformed gamma-glutamyl transferase, and low-density lipoprotein cholesterol.

ALT, alanine aminotransferase.

*Quartile: Q1, 1st quartile; Q2, 2nd quartile; Q3, 3rd quartile; Q4, 4th quartile. 
tendency as the serum ALT quartile increased ( $\mathrm{P}$ for trend $<0.05$ ).

\section{DISCUSSION}

It has been reported that increased triglyceride, total cholesterol, and LDL cholesterol levels; decreased HDL cholesterol levels; obesity; DM; smoking; HTN; and a family history of CHD are risk factors for CHD. ${ }^{15,16)}$ Our purpose was to determine the predictive value of ALT for the risk of $\mathrm{CHD}$ in a Korean population without DM or CHD using FRS modified by the NCEP ATP III guidelines.

Several studies have shown that NAFLD is associated with components of MetS and type $2 \mathrm{DM}^{2,10,17)}$ Fatty liver is regarded as the hepatic component of MetS. ${ }^{8)}$ Some studies have reported that NAFLD is an independent risk factor or CHD. ${ }^{12,13)}$ Patients with NAFLD are reported to have shorter survival time with higher CHD-related mortality. ${ }^{18,19)}$ ALT is a marker of NAFLD. ${ }^{2,17)}$ Targher and Day $^{20)}$ have shown that ALT is independently associated with CHD and that ALT could also be an independent predictor of $\mathrm{CHD} .^{21)}$

In this cross-sectional study, we found that ALT level was positively associated with 10-year CHD risk in Korean adults, using the FRS calculated with the NCEP ATP III algorithm. FRS is one of the scoring systems used to determine individual risk of developing CHD. We can estimate the probability that a person might develop CHD within 10-30 years through cardiovascular risk scoring systems. Higher quartiles of serum ALT level were found to be significantly associated with higher risk of CHD. These positive relationships remained significant after adjusting for established cardiovascular risk factors such as BMI, logtransformed GGT, alcohol consumption, and LDL cholesterol. When the quartile of serum ALT activity was increased, the OR of 10-year $\mathrm{CHD}$ risk prediction was also increased in a dose-dependent manner. In addition, the upper cut-off of third-quartile ALT level was defined as $\leq 24 \mathrm{IU} / \mathrm{L}$. This ALT level is considered normal according to most medical laboratories. The observation that the OR of intermediate or greater risk of 10-year CHD prediction using the ALT quartile was significantly increased, even though the ALT was within the normal reference range, suggests that ALT could be regarded as a risk stratification marker of FRS $\geq 10 \%$.

There are some possible mechanisms for the elevated ALT as a predictor of CHD. First, liver transaminase might be a marker of abnormal lipoprotein metabolism, ${ }^{22)}$ as it can induce accumulation of triglyceride-rich lipoproteins in the circulation. ${ }^{23)}$ In our study, we found that the group with higher quartiles of serum ALT had significantly higher triglyceride and LDL cholesterol levels, which can increase the risk of developing CHD. ${ }^{24)}$ Conversely, serum ALT was negatively correlated with HDL cholesterol level, a well-known negative risk factor for CHD. Second, high C-reactive protein levels in subjects with elevated ALT levels indicated that a systemic and hepatic inflammatory state might be associated with the development of coronary atherosclerosis and high risk of $\mathrm{CHD} .^{25)} \mathrm{A}$ review study reported that adipose tissue can promote the development of systemic and chronic low-grade inflammation. ${ }^{26)}$ The presence of a chronic inflammatory response along with metabolically harmful factors released by adipose tissue into the circulation is associated with accelerated atherosclerosis. ${ }^{12,13)}$ In a systemic inflammatory state, reactive oxygen species may induce the liver to produce cytokines such as tumor necrosis factor-alpha and interleukin-6. ${ }^{27,28)}$ These cytokines can cause hepatic fibrosis and inflammation, which may be associated with elevated ALT levels. ${ }^{13)}$ In our study, those with higher quartiles of serum ALT level had a significantly higher BMI than those with lower quartiles of serum ALT level. Thus, those with higher quartiles of BMI might have more adipose tissue that can induce an inflammatory state and accelerate atherosclerosis in coronary arteries.

Our results indicated that ALT was potentially a predictor of coronary atherosclerosis. Lifestyle modifications such as exercise and treatment with lipid-lowering agents might improve liver steatosis, thus slowing down the process of atherosclerosis and preventing CHD. Therefore, it might be appropriate to normalize and monitor transaminase levels using liver function tests in subjects with elevated ALT levels.

Bruckert et al. ${ }^{29)}$ found that elevated ALT was associated with CHD risk factors, including elevated $\mathrm{BP}$, total cholesterol, and triglyceride concentrations in 8,501 hyperlipidemic subjects. Schindhelm et al. ${ }^{13)}$ found that ALT predicts CHD events independent of traditional cardiovascular disease risk factors or components of MetS in 3,552 diabetic subjects. Shen et al. ${ }^{24)}$ found that serum ALT may serve as an independent predictor of CHD for early diagnosis and prevention in 610 inpatients at Shanghai Tenth People's Hospital. However, these studies were conducted among unhealthy subjects. On the other hand, our study included Korean adults without DM or CHD and demonstrated that ALT had predictive value for CHD. To the best of our knowledge, this is the first study to demonstrate the predictive value of ALT for CHD in the Korean population.

The strength of this study was that a broad range of individuals from the general population were included and selected randomly. Thus, it is rational to generalize the results to the Korean population. Moreover, we employed NCEP ATP III guidelines to compute the 10-year CHD risk.

This study has several limitations. First, this was a cross-sectional study and only included Koreans. Therefore, it cannot represent the general population of other countries. In addition, FRS might overestimate CHD risk in Asian-Pacific populations such as Japanese and Chinese. However, there is currently no other reliable or validated CHD risk assessment tool for Koreans. Thus, further longitudinal cohort studies are required to achieve the most accurate result to determine the predictive value of biomarkers for increased risk of CHD in the Korean population.

In conclusion, elevated serum ALT was significantly correlated with 10-year CHD risk in Korean adults using FRS calculated with NCEP ATP III guidelines. Therefore, serum ALT could be a useful tool as an additional marker in the prediction of CHD risk in Koreans. Further longitudinal cohort studies are required to achieve the most accurate results. 


\section{CONFLICT OF INTEREST}

No potential conflict of interest relevant to this article was reported.

\section{REFERENCES}

1. Price C, Alberti K. Biochemical assessment of liver function. In: Wright $\mathrm{R}$, editor. Liver and biliary diseases: pathophysiology, diagnosis, management. London: W.B. Saunders; 1979. p. 381-416.

2. Torres DM, Harrison SA. NAFLD: predictive value of ALT levels for NASH and advanced fibrosis. Nat Rev Gastroenterol Hepatol 2013;10: 510-1.

3. Cao W, Zhao C, Shen C, Wang Y. Cytokeratin 18, alanine aminotransferase, platelets and triglycerides predict the presence of nonalcoholic steatohepatitis. PLoS One 2013;8:e82092.

4. Carobene A, Braga F, Roraas T, Sandberg S, Bartlett WA. A systematic review of data on biological variation for alanine aminotransferase, aspartate aminotransferase and $\gamma$-glutamyl transferase. Clin Chem Lab Med 2013;51:1997-2007.

5. Gurung RB, Purbe B, Gyawali P, Risal P. The ratio of aspartate aminotransferase to alanine aminotransferase (AST/ALT): the correlation of value with underlying severity of alcoholic liver disease. Kathmandu Univ Med J (KUMJ) 2013;11:233-6.

6. Marchesini G, Brizi M, Bianchi G, Tomassetti S, Bugianesi E, Lenzi M, et al. Nonalcoholic fatty liver disease: a feature of the metabolic syndrome. Diabetes 2001;50:1844-50.

7. Westerbacka J, Corner A, Tiikkainen M, Tamminen M, Vehkavaara S, Hakkinen AM, et al. Women and men have similar amounts of liver and intra-abdominal fat, despite more subcutaneous fat in women: implications for sex differences in markers of cardiovascular risk. Diabetologia 2004;47:1360-9.

8. Cortez-Pinto H, Camilo ME, Baptista A, De Oliveira AG, De Moura MC. Non-alcoholic fatty liver: another feature of the metabolic syndrome? Clin Nutr 1999;18:353-8.

9. Knobler H, Schattner A, Zhornicki T, Malnick SD, Keter D, Sokolovskaya N, et al. Fatty liver: an additional and treatable feature of the insulin resistance syndrome. QJM 1999;92:73-9.

10. Gupte P, Amarapurkar D, Agal S, Baijal R, Kulshrestha P, Pramanik S, et al. Non-alcoholic steatohepatitis in type 2 diabetes mellitus. J Gastroenterol Hepatol 2004;19:854-8.

11. Hanley AJ, Williams K, Festa A, Wagenknecht LE, D'Agostino RB Jr, Haffner SM. Liver markers and development of the metabolic syndrome: the insulin resistance atherosclerosis study. Diabetes 2005;54: 3140-7.

12. Dekker JM, Girman C, Rhodes T, Nijpels G, Stehouwer CD, Bouter LM, et al. Metabolic syndrome and 10-year cardiovascular disease risk in the Hoorn Study. Circulation 2005;112:666-73.

13. Schindhelm RK, Dekker JM, Nijpels G, Bouter LM, Stehouwer CD, Heine RJ, et al. Alanine aminotransferase predicts coronary heart disease events: a 10-year follow-up of the Hoorn Study. Atherosclerosis 2007; 191:391-6.

14. Greenfield TK. Ways of measuring drinking patterns and the differ- ence they make: experience with graduated frequencies. J Subst Abuse 2000;12:33-49.

15. Anthony D, George P, Eaton CB. Cardiac risk factors: biomarkers and genetic tests to determine cardiovascular risk. FP Essent 2014;421:115.

16. Dalen JE, Alpert JS, Goldberg RJ, Weinstein RS. The epidemic of the 20(th) century: coronary heart disease. Am J Med 2014;127:807-12.

17. Verma S, Jensen D, Hart J, Mohanty SR. Predictive value of ALT levels for non-alcoholic steatohepatitis (NASH) and advanced fibrosis in non-alcoholic fatty liver disease (NAFLD). Liver Int 2013;33:1398-405.

18. Lu H, Zeng L, Liang B, Shu X, Xie D. High prevalence of coronary heart disease in type 2 diabetic patients with non-alcoholic fatty liver disease. Arch Med Res 2009;40:571-5.

19. Gaggini M, Morelli M, Buzzigoli E, DeFronzo RA, Bugianesi E, Gastaldelli A. Non-alcoholic fatty liver disease (NAFLD) and its connection with insulin resistance, dyslipidemia, atherosclerosis and coronary heart disease. Nutrients 2013;5:1544-60.

20. Targher G, Day CP. Liver enzymes, nonalcoholic fatty liver disease, and incident cardiovascular disease. Hepatology 2011;53:375.

21. Lonardo A, Sookoian S, Chonchol M, Loria P, Targher G. Cardiovascular and systemic risk in nonalcoholic fatty liver disease: atherosclerosis as a major player in the natural course of NAFLD. Curr Pharm Des 2013;19:5177-92.

22. Charlton M, Sreekumar R, Rasmussen D, Lindor K, Nair KS. Apolipoprotein synthesis in nonalcoholic steatohepatitis. Hepatology 2002;35: 898-904.

23. Gianturco SH, Bradley WA. Lipoprotein-mediated cellular mechanisms for atherogenesis in hypertriglyceridemia. Semin Thromb Hemost 1988;14:165-9.

24. Shen J, Zhang J, Wen J, Ming Q, Zhang J, Xu Y. Correlation of serum alanine aminotransferase and aspartate aminotransferase with coronary heart disease. Int J Clin Exp Med 2015;8:4399-404.

25. Kerner A, Avizohar O, Sella R, Bartha P, Zinder O, Markiewicz W, et al. Association between elevated liver enzymes and C-reactive protein: possible hepatic contribution to systemic inflammation in the metabolic syndrome. Arterioscler Thromb Vasc Biol 2005;25:193-7.

26. Cinkajzlova A, Mraz M, Haluzik M. Lymphocytes and macrophages in adipose tissue in obesity: markers or makers of subclinical inflammation? Protoplasma 2017;254:1219-32. https://doi.org/10.1007/s00709017-1082-3.

27. Ioannou GN. Implications of elevated serum alanine aminotransferase levels: think outside the liver. Gastroenterology 2008;135:1851-4.

28. Yamada J, Tomiyama H, Yambe M, Koji Y, Motobe K, Shiina K, et al. Elevated serum levels of alanine aminotransferase and gamma glutamyltransferase are markers of inflammation and oxidative stress independent of the metabolic syndrome. Atherosclerosis 2006;189:198205.

29. Bruckert E, Giral P, Ratziu V, Poynard T, Chapman MJ, Opolon P, et al. A constellation of cardiovascular risk factors is associated with hepatic enzyme elevation in hyperlipidemic patients. Metabolism 2002;51: 1071-6. 